\title{
Suchtmedizinische Kurzinterventionen in der Grundversorgung
}

\author{
Brief Interventions for Substance-Related Disorders in Primary Care
}

\section{Zusammenfassung}

Kurzinterventionen basieren in der Suchtmedizin meist auf kognitiv-behavioralen oder auf motivationalen Konzepten. Nach einer Erläuterung der allgemeinen Prinzipien werden beispielhaft einzelne bewährte Settings vorgestellt.

Kurzinterventionen vermindern zum Teil nachhaltig den Konsum psychotroper Substanzen. Obwohl ihre Effektstärken weit geringer als die von längerfristigen Behandlungen sind, empfiehlt sich ihr Einsatz insbesondere im Bereich der Primärversorgung, da mit relativ geringem Zeitaufwand eine große Zahl von Personen mit schädlichem oder mit abhängigem Konsum behandelt werden kann. Ferner erhöhen Kurzinterventionen die Bereitschaft, weiterführende Hilfen in Anspruch zu nehmen.

\section{Schliuisselwörter}

Substanzkonsum - Kurzintervention · Suchtmedizinische Empfehlung · Motivation

\section{Abstract}

Minimal and brief interventions for individuals with substancerelated problems are mostly based on cognitive-behavioral or on motivational concepts. General approaches of screening and individual assessment are presented. Some examples of effective settings are outlined.

Brief interventions decrease substance use over a period up to two years. The effect is smaller than in intensive treatment. However, in primary care minimal or brief interventions are more feasible because far less time for training and treatment is needed. Furthermore they increase the readiness to join settings of more intensive therapy.

\section{Key words}

Substance abuse $\cdot$ brief intervention $\cdot$ brief advice $\cdot$ motivation
Unter Kurzinterventionen werden nach Babor [1] alle suchtmedizinischen Behandlungsformen verstanden, die sich auf maximal drei Behandlungstermine beziehen. Die medizinische Grundversorgung ist ein günstiger Ort zur Durchführung solcher Interventionen, da die Bevölkerung hier gegenwärtig einen niederschwelligen Zugang hat. Zum Beispiel stellen sich mindestens 80\% aller Menschen mit einer Alkoholproblematik innerhalb eines Jahres bei niedergelassenen Ärzten vor [2-4].

\section{Elemente von Kurzinterventionen}

\section{Die Fallidentifikation}

In wissenschaftlichen Studien zur Wirksamkeit suchtmedizinischer Kurzinterventionen werden oft Screeningtests zur Identifikation geeigneter Fälle eingesetzt. Sie erlauben eine Einschätzung der Prävalenz und manchmal auch des Schweregrades einer substanzbedingten Störung in der Interventionsgruppe. Ein positi- 
ves Ergebnis im Screeningtest erleichtert es ferner, den Patienten für eine Teilnahme an der Kurzintervention zu gewinnen.

Aushändigung und Auswertung zum Beispiel des FagerströmTests [5] unter Rauchern oder des AUDIT [6] beziehungsweise des LAST [7] unter Alkoholkonsumenten durch die Arzthelferin stellen einen geringen Arbeitsaufwand dar. Selbstverständlich kann eine effiziente Fallidentifikation in der hausärztlichen Alltagsarbeit auch unsystematisch erfolgen.

\section{Die Berücksichtigung der Patientenmotivation}

Vor suchtmedizinischen Behandlungsmaßnahmen ist die aktuelle Motivationslage des Patienten einzuschätzen. Bei diesem im englischsprachigen Raum Assessment genannten Vorgang wird auf die von Prochaska und DiClemente [8, 9] für Abhängigkeitskranke beschriebenen Stadien der Motivation zurückgegriffen.

Danach hat jeder Abhängigkeitskranke die Möglichkeit, zwischen Phasen des Konsums und der Abstinenz zu wechseln.

Beim Konsum werden drei Motivationsphasen unterschieden. Der Zustand des unreflektierten Konsums wird als Präkontemplation bezeichnet. Mit dieser Wortwahl bringen die Autoren zum Ausdruck, dass auch bei jahrelangem, ununterbrochenem und unreflektiertem Konsum jederzeit der Übergang in die folgende Kontemplationsphase möglich ist. Sie wird erreicht, indem der Patient zunehmend negative Folgen seines Konsums beachtet. Die sich anschließende Phase der Entscheidung für eine konkrete Verhaltensänderung in Richtung auf Abstinenz ist zeitlich oft recht kurz.

Die nachfolgende Abstinenz ist insbesondere bei Substanzen mit hohem Suchtpotenzial wie zum Beispiel beim Tabak meist zunächst nicht dauerhaft. Nach einem Rückfall steht es dem Betroffenen frei, zunächst unreflektiert zu konsumieren oder aber rasch mit der Kontemplation von Suchtfolgeschäden und einer entsprechenden Entscheidung die nächste Abstinenzphase vorzubereiten.

Wird im Vorfeld zum Beispiel einer Intervention in Bezug auf einen offensichtlich schädlichen Alkoholkonsum festgestellt, dass der Patient aktuell direkt vor einer Abstinenzentscheidung steht, darf das Ergebnis dieses Assessments nicht auf den Konsum anderer psychotroper Substanzen übertragen werden. Der gleiche Patient kann bezüglich seines Tabakkonsums im Stadium der Präkontemplation sein.

\section{Die korrekte ärztliche Zielsetzung}

Aus der Einschätzung der aktuellen Motivationslage ergibt sich die grundsätzliche ärztliche Zielrichtung für eine Kurzintervention. Bei aktuellem Konsum wird in der Regel jeweils zunächst die nächsthöhere Motivationsphase angestrebt, indem die Präkontemplation in die Kontemplation und die Kontemplation in die Entscheidungsphase überführt werden. Befindet sich der Patient bereits in der Phase der Entscheidung, wird seine Planung im Rahmen der Kurzintervention konkretisiert und operationalisiert [10]. Bei aktueller Abstinenz wird rückfallpräventiv gearbeitet.
Das kleinschrittige Vorgehen bei konsumierenden Abhängigkeitskranken widerspricht der unter Ärzten verbreiteten Ungeduld, abhängigen Konsum rasch zu beenden. Hierzu ist anzumerken, dass der abhängige Konsum in den meisten Fällen schon über eine längere Zeit besteht und dass der Patient in der Regel bereits in eigener Regie vergeblich versucht hat, seinen Konsum zu begrenzen. Das ungeduldige Einfordern von Abstinenz unterstützt das weit verbreitete Klischee vom autoritär und moralisierend auftretenden Arzt, der unabhängig vom Lebensumfeld des Patienten eine Leistung fordert, die dieser offensichtlich bisher nicht erbringen konnte. Ein solches ärztliches Verhalten kann dem Patienten Angst machen und dessen Widerstand und NonCompliance fördern. Einzelne Ärzte schaffen sich auf diese Weise eine Klientel ungenügend kooperierender Patienten, die dann zur Rechtfertigung der Annahme herangezogen wird, die Arbeit mit Abhängigkeitskranken sei frustran [11,12].

Wenn lediglich kleinere Schritte in Richtung auf Abstinenz nahegelegt werden, wird der Arzt eher als verständnisvoll und empathisch wahrgenommen. Sofern der Patient dann ein rascheres Vorgehen fordert, liegt die Initiative auf der richtigen Seite des Patient-Arzt-Verhältnisses. Sie kann professionell aufgegriffen und professionell unterstützt werden.

\section{Beispiele suchtmedizinischer Kurzinterventionen}

Zahlreiche Formen von Kurzinterventionen bei riskantem, schädlichem oder abhängigem Substanzkonsum sind publiziert. Die Mehrzahl bezieht sich auf Alkohol oder Tabak [13-16]. Der erforderliche Schulungsaufwand für den Therapeuten streut je nach Verfahren zwischen mehrwöchigen Seminaren und dem Durchlesen eines Manuals. Der Zeitaufwand pro Patient variiert zwischen dreimal einer Stunde und einmalig drei bis fünf Minuten $[1,16]$.

Die zugrunde liegenden Behandlungsansätze sind ganz überwiegend kognitiv-behavioral oder motivational. Erstere identifizieren anhand von Verhaltensanalysen und oft standardisierten Selbstbeobachtungen Risiken und erarbeiten durch oft manualisierte Interventionen alternative Kognitionen und Verhaltensweisen. Letztere werden bei Alkoholproblemen nach Miller [13, 17, 18] mit dem Akronym FRAMES umschrieben:

- Feedback (wertungsfreie Rückmeldung zu Suchtfolgeschäden)

- Responsibility (Stärkung der Eigenverantwortlichkeit)

- Advice (Ratschlag zur Schadens- oder Risikoverminderung)

- Menue (Auswahlmöglichkeit zwischen verschiedenen Verhaltensweisen)

- Empathy (empathische Haltung des Behandelnden)

- Self-Efficacy (Stärkung der Selbstwirksamkeitserwartung des Patienten)

Im Folgenden werden motivationale Ansätze, ein Setting, das beide Therapieansätze miteinander kombiniert, und abschließend etwas ausführlicher eine motivationale Minimalintervention (suchtmedizinische Empfehlung) vorgestellt.

\section{Klassisches Motivational Interviewing (MI)}

MI wurde in Form von Sequenzen ambulanter Gespräche zur Förderung der Abstinenz- und der Behandlungsmotivation bei Alkoholkranken mit hohem Widerstand und mit geringer Krankheits- 
einsicht entwickelt. Der Patient bestimmt zunächst das Gesprächsthema und erhält Unterstützung, seine Sichtweisen und Anliegen zu vermitteln.

Beim MI handelt es sich insofern um eine direktive Form der Gesprächsführung, als durch eine überschaubare Anzahl genau definierter Interventionen der Patient angeregt wird, auch über subjektive Abstinenzmotive zu sprechen. Die Motivation erfolgt vorrangig dadurch, dass der Patient sich selbst ausführlich über die für ihn relevanten Nachteile des Konsums und die entsprechenden Vorteile der Konsumreduktion sprechen hört. Meist bei der zweiten oder der dritten Sitzung macht der Patient von sich aus Vorschläge zu Verhaltensänderungen in Richtung Abstinenz. Diese werden vom Therapeuten hinterfragt und dadurch vom Patienten konkretisiert und bekräftigt.

Nur bei durchgängiger Bagatellisierung oder Verleugnung jedweder substanzbedingter Probleme wird der Patient gefragt, ob er an einer fachlichen Einschätzung seiner Situation interessiert sei. Sofern dies der Fall ist, erhält der Patient Informationen über objektive Suchtfolgeschäden, die für ihn eingetreten oder aktuell zu erwarten sind. Dabei enthält sich der Therapeut in Wortwahl und Tonfall jedweder Konfrontation oder Vorwurfshaltung, da dies dem Behandlungserfolg abträglich ist [19]. Für Streitgespräche zum Beispiel über das etwaige Bestehen einer Abhängigkeit steht der Therapeut nicht zur Verfügung. Er schafft stattdessen mit aufmerksamer Empathie optimale Voraussetzungen dafür, dass der Patient sich zunehmend kompetent fühlt, einen ernsthaften Abstinenzversuch zu wagen [20, 21]. Er unterstützt den Patienten bei der Formulierung und Erreichung relevanter Ziele.

Mit diesem Konzept der geduldigen Entwicklung nachhaltiger Patientenmotivation im Verlauf mehrerer Gesprächstermine geht das klassische MI vom Behandlungsumfang oft über den Rahmen einer Kurzintervention hinaus [18].

\section{Brief Motivational Interviewing}

Da der Umfang der erforderlichen therapeutischen Ausbildung und der für die einzelne Behandlung nötige Zeitaufwand beim klassischen MI seine Anwendung in der Basisversorgung erschwert, entwickelten Rollnick und Kollegen [22] eine Kurzform. Diese als Brief Motivational Interviewing bezeichnete Vorgehensweise übernimmt die oben skizzierte empathische Grundhaltung des Therapeuten und wesentliche Einzelinterventionen aus dem klassischen MI. Sie beschränkt sich jedoch auf einen einzelnen Gesprächskontakt mit dem Patienten. Für ein solches Brief-MI erlernen die Therapeuten in einem vorgeschalteten Training acht definierte Eröffnungsstrategien, aus denen sie je nach Ausgangslage des Patienten eine auswählen. Innerhalb von 30 bis 40 Minuten wird mit dem Ziel der Trinkmengenreduktion gezielt auf die Motivation des Patienten Einfluss genommen.

Heather et al. [23] untersuchten 174 als „heavy drinkers“ identifizierte männliche Patienten somatischer Krankenhausabteilungen, deren Alkoholproblem entweder im üblichen Rahmen der Krankenhausstation, mit einer kognitiv-behavioralen Kurzintervention oder mit Brief-MI behandelt worden war. Bei der 6-Monats-Katamnese erwiesen sich beide Kurzinterventionsformen als der Standardbehandlung deutlich überlegen. Primär unmoti- vierte Patienten zeigten nach dem Brief-MI eine stärkere Reduktion der Trinkmenge.

\section{Brief Alcohol Screening and Intervention for College Students (BASICS)}

Diese insgesamt zwei bis drei therapeutische Sitzungen von bis zu 50 Minuten umfassende Kurzintervention integriert motivationale und kognitiv-behaviorale Konzepte.

Der motivationale Anteil entspricht weit gehend dem „Drinkers Check-up“, bei dem im Stil des MI nach einer umfangreicheren diagnostischen Erhebung u. a. die Trinkmengen und -gewohnheiten bei einer zweiten Sitzung in grafischer Form im Sinne vorwurfsfreier Information mit Durchschnittswerten einer Referenzstichprobe verglichen werden. Dem Patienten wird es überlassen, aus der erhaltenen Information Schlüsse zu ziehen. In randomisierten Studien zeigte sich diese motivationale Intervention als mit unterschiedlicher Zeitstabilität einer Standardbehandlung überlegen $[24,25]$.

BASICS fügt zwischen die beiden Sitzungen dieser motivationalen Intervention eine vollständige Erhebung der positiven und der negativen Trinkerwartungen und das Führen eines standardisierten Trinkprotokolls über einen Zeitraum von ein bis zwei Wochen ein.

Borsari und Carey [26] fanden in einer randomisierten kontrollierten Studie an 60 Studenten sechs Wochen nach BASICS sowohl eine Abnahme der Häufigkeit des Alkoholkonsums wie auch eine Reduktion exzessiver Alkoholaufnahmen. In einer anderen randomisierten Studie an 348 Studenten mit zumindest riskantem Konsum ließ sich zwei Jahre nach der Intervention nicht nur eine anhaltende Reduktion der Trinkmenge, sondern auch der gesundheitlichen sowie der sozialen Probleme nachweisen [27].

\section{Die suchtmedizinische Empfehlung}

Sofern aktuell unbehandelter schädlicher oder abhängiger Konsum bei Patienten deutlich wird, im ärztlichen Arbeitsalltag jedoch keine Zeit für eine der oben genannten Interventionen zur Verfügung steht, liegt eine kurz gefasste ärztliche Empfehlung nahe. Regelmäßiger Tabakkonsum ist in jedem Fall zumindest schädlich.

Vor dem Aussprechen der Empfehlung wird der Patient gefragt, ob er bezüglich seines aktuellen Konsums an einem ärztlichen Rat interessiert sei.

Einzelne Patienten verneinen dies mit dem Hinweis, diesen Ratschlag sich bereits denken zu können. In dieser Situation empfiehlt es sich, den Patienten zu bitten, seine Vermutung auszusprechen. Sofern der Patient dabei grob vom Inhalt der ärztlichen Empfehlung abweicht, wird dies mitgeteilt und zur Ausgangsfrage zurückgekehrt. Falls die Vermutung des Patienten im Wesentlichen zutrifft, wird dies anerkannt und die Intervention ist erfolgreich abgeschlossen.

Nahezu alle Patienten werden dem Arzt die Erlaubnis erteilen, eine Empfehlung zu geben. Sofern dabei lediglich sachte genickt wird, ist es durchaus sinnvoll, die Frage zu wiederholen. Es ist 
wirksam, wenn der Patient die eigene Stimme hört, die den Arzt zu einem Ratschlag auffordert. Der Patient bekundet damit Interesse an einer möglichen Verbesserung seiner Situation. Die nachfolgende ärztliche Empfehlung wird vom Patienten weniger als fremd oder als unverfroren angesehen.

Der zweite Teil umfasst die ärztliche Empfehlung. Sie sollte kurz und gut verständlich sein. Sie sollte die aktuellen Möglichkeiten des Patienten respektieren und, wie oben angegeben, ein konkretes, für den Patienten voraussichtlich subjektiv erreichbares (Teil-)Ziel benennen. Im Zweifelsfall ist das bescheidenere Ziel vorzuschlagen.

Sofern der Patient auf den Ratschlag mit Rechtfertigungen oder Argumentationen reagiert, wird hierauf nicht eingegangen. Gegebenenfalls wird daran erinnert, dass lediglich die Übermittlung eines Ratschlags verabredet war.

Sofern der Patient konstruktiv auf den Ratschlag eingeht, ist ihm ein Termin zu einer ausführlicheren Beratung anzubieten, die selbstverständlich auch in einer entsprechenden Beratungsstelle oder einer suchtmedizinischen Ambulanz beziehungsweise Schwerpunktpraxis erfolgen kann (Abb.1 nach [28]).

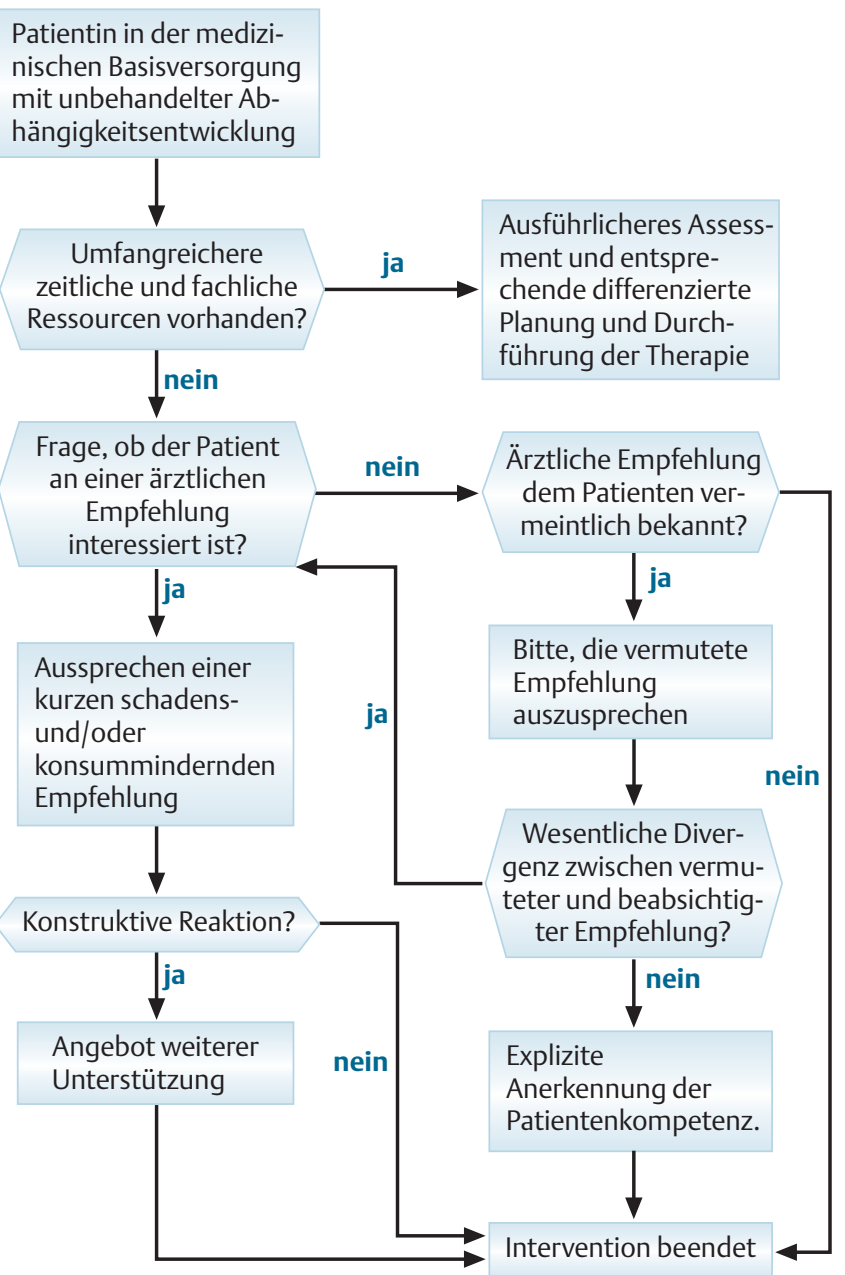

Abb. 1 Klinischer Algorithmus nach Lorenz und Ollenschläger [28] zur Indikation und Durchführung einer suchtmedizinischen Empfehlung.

\section{Differenzialindikation verschiedener Kurzinterventionen}

Grundsätzlich ist innerhalb jeder ärztlichen Behandlung die Durchführung einer suchtmedizinischen Intervention indiziert, wenn ein unbehandelter schädlicher oder abhängiger Substanzkonsum deutlich wird. Auch bei einem problematischen Konsum kann bereits interveniert werden. Es wäre falsch, den Patienten so lange unbehandelt zu lassen, bis er von sich aus um Behandlung bittet. Die Wirksamkeit von Kurzinterventionen ist auch bei Personen, die nicht Therapie nachfragen, erwiesen [29]. Ferner ist wahrscheinlich, dass die Erfolge von Kurzinterventionen in diesem Kollektiv zeitstabiler sind, wenn sie in früheren Stadien der Abhängigkeitsentwicklung vorgenommen werden [29].

Sofern die Vorstellung auf externen Druck zum Beispiel durch einen Familienangehörigen, das Jugendamt oder den Arbeitgeber zustande kommt und eine Bagatellisierung oder sogar eine Verleugnung der konsumbezogenen Probleme zu erwarten ist, stellt klassisches Motivational Interviewing eine besonders gut geeignete Interventionsform dar. Sie erfordert relativ viel Zeit und eine umfassendere Fortbildung des Therapeuten.

Wenn eine dieser beiden Voraussetzungen nicht gegeben ist, empfiehlt sich eine der weiteren oben skizzierten strukturierten Kurzinterventionen. Diese sind bei geringerem Widerstandsniveau des Patienten primär indiziert.

Sofern für eine solche Intervention Zeit oder praktische Erfahrung fehlen, sollte in jedem Fall eine ärztliche Empfehlung angeboten werden. Abb. 2 fasst die Differenzialindikation suchtmedizinischer Kurzinterventionen zusammen.

\section{Von der Kurzintervention zur suchtmedizinischen Behandlung}

Trotz ihrer nachgewiesenen Wirksamkeit sind suchtmedizinische Kurzinterventionen [z. B. 13, 16, 29] allein in der Regel keine ausreichende Behandlungsmaßnahme. So ist anlässlich der Intervention nach Möglichkeit weiterführende suchtmedizinische Behandlung anzubieten. Allein das Angebot, bei einem Folgetermin auf das angesprochene Problemfeld gegebenenfalls

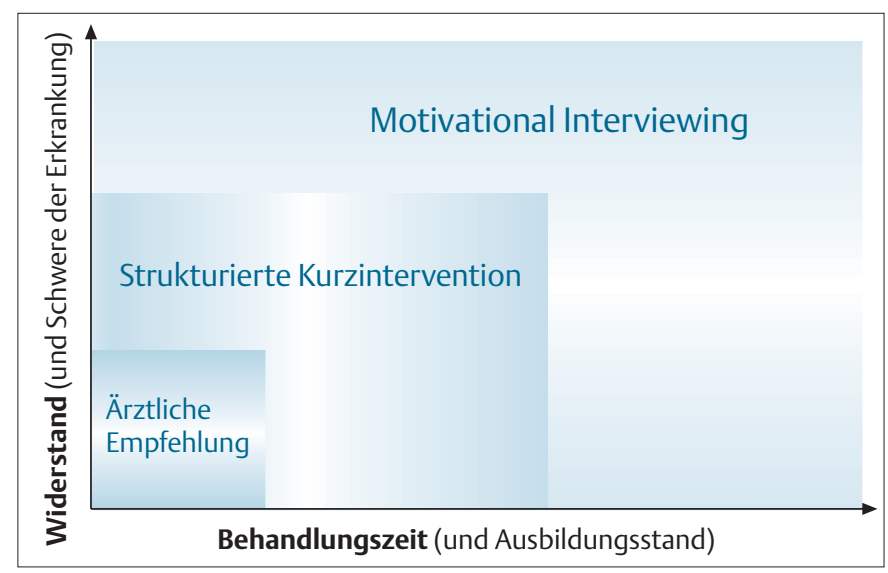

Abb. 2 Differenzialindikation verschiedener suchtmedizinischer Interventionen bezüglich des Widerstandsniveaus des Patienten beziehungsweise der Schwere der Abhängigkeitserkrankung. 
zurückzukommen, ist hier hilfreich. Über weiterführende Hilfen innerhalb und gegebenenfalls auch außerhalb des Suchthilfesystems ist je nach Problemlage zu informieren. Die meisten regional arbeitenden Suchtberatungsstellen, suchtmedizinischen Krankenhausabteilungen und Schwerpunktpraxen stellen auf Anfrage gern schriftliches Informationsmaterial zur Verfügung, das dem Patienten mitgegeben werden kann.

Sofern die Indikation für eine suchtmedizinische Medikation besteht, sollte dies dem Patienten vermittelt werden. Nikotinpflaster und/oder -kaugummi und/oder Nasalspray sind in der Regel vom Patienten selbst zu finanzieren. Eine entsprechende Beratung durch den Arzt erleichtert dem Patienten den Schritt, diese wirksame Unterstützung tatsächlich in Anspruch zu nehmen.

Bei konsumierenden Alkoholabhängigen kann im Rahmen eines ambulanten Entzugs ggf. Carbamazepin rezeptiert werden. Nach abgeschlossenem Entzug kann die Gabe von Acamprosat indiziert sein. Für Heroinabhängige sei auf die Möglichkeiten einer Substitution zum Beispiel mit Methadon oder Buprenorphin und auf die in Deutschland bisher wenig genutzte Möglichkeit der Abstinenzsicherung durch Nemexin hingewiesen.

Diesen verschiedenen Medikationen ist gemeinsam, dass sie neben der pharmakologischen Wirkung die Möglichkeit eröffnen, die Beziehung zwischen dem Abhängigen und seinem behandelnden Arzt zu verstetigen und gegenseitiges Vertrauen wachsen zu lassen, das eine Voraussetzung für weiter reichende Behandlungsformen und für eine Vorbereitung auf eine etwaige medizinische Rehabilitation darstellt.

\section{Häufige Fehler}

Das oben geschilderte kleinschrittige und geduldige Vorgehen bei der Zielsetzung suchtmedizinischer Kurzinterventionen darf keinesfalls verwechselt werden mit einer Verharmlosung der Abhängigkeitsentwicklung oder der Suchtfolgeschäden. $\mathrm{Zu}$ Recht wird patientenseitig vom Arzt erwartet, dass dieser die Krankheitsentwicklung in jedem Fall ernst nimmt.

Nachteilig für die suchtmedizinische Behandlung sind Verbrüderungen zwischen Arzt und Patient, bei denen die aktuellen Konsummengen oder -muster des Patienten mit denen des Arztes verglichen werden. Den Vergleich zu Konsummustern anderer Personen nimmt der Patient ohnehin in seinem privaten Umfeld vor. Den Arzt nimmt er in Anspruch, weil er dort ein tiefer gehendes Fachwissen und kompetentere Unterstützung seiner Anliegen erwartet.

\section{Literatur}

${ }^{1}$ Babor TF. Avoiding the horrid and beastly sin of drunkeness. Does Dissuasion make a difference? Journal of Consulting and Clinical Psychology 1994; 62: 1127-1140

2 Wienberg G. Die vergessene Mehrheit. In: Wienberg G (Hrsg). Bonn Psychiatrie-Verlag, 1992

${ }^{3}$ Wienberg G. Die „vergessene Mehrheit“ heute - Teil IV: Zur Situation in der medizinischen Primärversorgung. In: Wienberg G, Driessen M
(Hrsg). Auf dem Weg zur vergessenen Mehrheit. Bonn: PsychiatrieVerlag, 2001

${ }^{4}$ Rumpf HJ, Meyer C, Hapke U et al. Inanspruchnahme suchtspezifischer Hilfen von Alkoholabhängigen und -mißbrauchern: Ergebnisse der TACOS-Bevölkerungsstudie. Sucht 2000; 46: 9-17

${ }^{5}$ Fagerström KO, Heatherton TF, Kozlowski LT. Nicotine addiction and its assessment. Ear, Nose Throat J 1991; 69 (11): 763 - 768

${ }^{6}$ Babor TF, de la Fuente JR, Saunders J et al. The alcohol use disorders identification test: Guidelines for use in primary health care. Geneva: World Health Organization, 1999

${ }^{7}$ Rumpf HJ, Hapke U, Hill A et al. Development of a screening questionnaire for the general hospital and general practices. Alcoholism: Clinical and Experimental Research 1997; 21: 894-898

8 Prochaska OJ, DiClemente CC. In search of how people change. Applications to addictive behaviours. Am Psychol 1992; 47: 1102 - 1114

${ }^{9}$ Prochaska JO, Velicer WF. The transtheoretical model of health behavior change. Am J Health Promot 1997; 12: 38 -48

${ }^{10}$ Miller WR. Motivation and treatment goals. Drugs and Society 1987; 1: $133-151$

${ }^{11}$ Grampp P. Motivation in der Suchttherapie. In: Fleischmann H, Klein HE (Hrsg). Behandlungsmotivation/Motivationsbehandlung, Suchtkranke im Psychiatrischen Krankenhaus. Freiburg: Lambertus, 1995

${ }^{12}$ Reymann G. Die Arzt-Patient Beziehung. In: Poehlke T, Flenker I, Follmann A, Rist F, Kremer G (Hrsg). Grundlagen der Behandlung. Berlin/ Heidelberg/New York: Springer, 2000; 169-178

13 Bien TH, Miller WR, Tonigan JS. Brief interventions for alcohol problems: a review. Addiction 1993; 88: 315 - 336

${ }^{14}$ Fiore MC, Bailey WC, Cohen SJ et al. Treating tobacco use and dependence. Clinical practice guideline. Rockville, MD: US Department of Health and Human Services. Am J Public Health Service 2000

15 Schmidt LG. Tabakabhängigkeit und ihre Behandlung. Dt Ärzteblatt 2001; 98: 1826-1833

${ }^{16}$ Silagy C. Physician advice for smoking cessation (Cochrane Review). The Cochrane Library, Issue 4. Oxford 2000

${ }^{17}$ Miller WR, Sanchez VC. Motivating young adults for treatment and lifestyle change. In: Howard GS, Nathan PE (Hrsg). Alcohol use and misuse by young adults. nnnnnnnnn: University of Notre Dame Press, 1994; $55-82$

${ }^{18}$ Demmel R. Motivational Interviewing: Ein Literaturüberblick. Sucht 2001; 47: $171-188$

${ }^{19}$ Miller WR, Benefield RG, Tonigan JS. Enhancing motivation for change in problem drinking: A controlled comparision of two therapist styles. Journal of Consulting and Clinical Psychology 1993; 61: 455-461

${ }^{20}$ Miller WR, Rollnick S. Motivational Interviewing. 2 ed. New York: Guilford, 2001

${ }^{21}$ Miller WR, Rollnick S. Motivierende Gesprächsführung (dt. Übersetzung der 1. englischsprachigen Auflage). Freiburg: Lambertus, 1999

22 Rollnick S, Heather N, Bell A. Negotiating behaviour change in medical settings: the development of brief motivational interviewing. Journal of Mental Health UK 1992; 1: 25-37

${ }^{23}$ Heather N, Rollnick S, Bell A et al. Effects of brief counselling among male heavy drinkers identified on general hospital wards. Drug and Alcohol Review 1996; 15: 29-38

${ }^{24}$ Bien TH, Miller WR, Boroughs JM. Motivational interviewing with alcohol outpatients. Behavioural and Cognitive Psychotherapy 1993; 21: $347-356$

25 Bombardier CH, Rimmele CT. Motivational interviewing to prevent alcohol abuse after traumatic brain injury: A case series. Rehabilitation Psychology 1999; 44: 52-67

${ }^{26}$ Borsari B, Carey KB. Effects of a brief motivational intervention with college student drinkers. Journal of Consulting and Clinical Psychology 2000; 68: $728-733$

${ }^{27}$ Marlatt GA, Baer JS, Kivlahan DR et al. Screening and brief intervention for high-risk college student drinkers: results form a 2-year follow-up assessment. Journal of Consultant and Clinical Psychology 1998; 66: $604-615$

${ }^{28}$ Lorenz W, Ollenschläger G. Das Leitlinien-Manual der Arbeitsgemeinschaft der Wissenschaftlichen Medizinischen Fachgesellschaften (AWMF) und der Ärztlichen Zentralstelle Qualitätssicherung (ÄZQ). ZaeFQ 2000; 94

${ }^{29}$ Moyer A, Finney JW, Swearingen CE et al. Brief interventions for alcohol problems: a meta-analytic review of controlled investigations in treatment-seeking and non-treatment-seeking populations. Addiction 2002; 97: 279-292 